\title{
Tiling theory applied to the surface structure of icosahedral AlPdMn quasicrystals.
}

\author{
Peter Kramer, Zorka Papadopolos, Harald Teuscher \\ Institut für Theoretische Physik der Universität Tübingen
}

19th November 2018

\begin{abstract}
.
Surfaces in i- $A l_{68} P d_{23} M n_{9}$ as observed with STM and LEED experiments show atomic terraces in a Fibonacci spacing. We analyze them in a bulk tiling model due to Elser which incorporates many experimental data. The model has dodecahedral Bergman clusters within an icosahedral tiling $\mathcal{T}^{*(2 F)}$ and is projected from the $6 D$ face-centered hypercubic lattice. We derive the occurrence and Fibonacci spacing of atomic planes perpendicular to any 5fold axis, compute the variation of planar atomic densities, and determine the (auto-) correlation functions. Upon interpreting the planes as terraces at the surface we find quantitative agreement with the STM experiments.
\end{abstract}

\section{Introduction.}

The bulk structure of the icosahedral phases i- $A l P d M n$, i- $A l F e C u$ and their modelling in terms of a $6 D$ description has been an active research field for more than one decade. From the many papers on this topic we mention only a few. Many more references are quoted in these publications. Katz and Gratias [7] derive from previous work for i- $A l F e C u$ a quasiperiodic network of atomic positions. It is generated by three basic atomic windows related to the $6 D$ hypercubic F-lattice. They examine carefully the interatomic distances. De Boissieu et al. |2 2] determine for i-AlPdMn from X-ray and neutron data in detail the decomposition of the atomic surfaces. All these models use the $6 D$ embedding, the parallel and the perpendicular space. Elser [4] generalizes and unifies both these models and interprets them in terms of clusters occupying the odd and even vertices of the icosahedral tiling 
related to the $6 D$ hypercubic P-lattice: The odd vertices form the centers of Bergman clusters, which then around the even vertices build up Mackay clusters. Additional atomic positions are related to this basic structure. The Elser model was actually created for the study of random tilings, but by construction admits a perfect tiling structure which then incorporates the main experimental data which have led to the models by Katz and Gratias and by de Boissieu et al. As shown in [11], the Elser model can be taken as a network of atomic positions in a tiling model, denoted by $\mathcal{T}^{*(2 F)}$ and related to the observed hypercubic F-lattice and -module. This tiling model will be used in what follows. Its composite atomic surfaces are closely related to those of the Katz-Gratias model [7].

The surface structure of i- $A l_{68} P d_{23} M n_{9}$ perpendicular to 5 fold axes has been explored by various groups. Schaub et al. 15 applied scanning tunneling microscopy (STM) and low-energy electron diffraction (LEED) to obtain atomic scale information of a sputtered and annealed surface. They observe a sequence of 11 atomically flat terraces. Two spacings of $(4.22 \pm 0.26)$ and $(6.78 \pm 0.24) \AA$ form a Fibonacci string of the type LLSLLSLSLL. Pentagonal holes of a single fixed orientation appear within these terraces. Gierer et al [5, 6] by dynamical LEED studies for a similarly prepared surface confirm the quasicrystalline structure. To interpret their data they perform dynamical diffraction calculations for assumed terminations of a model patch from the bulk model of de Boissieu et al. [2]. They find optimal agreement for Al-rich terminations of high atomic density. A study by Ebert et al. [3] of in-situ cleaved surfaces revealed terraces only after annealing of the initially rather rough surface.

For the theory of quasicrystals, the experiments raise the question what quasiperiodic repetition pattern and what structure variation within planes can be rigorously obtained from a bulk model of i- $A l_{68} P d_{23} M n_{9}$. In the present paper we address these questions in the description by the tiling model. We develop a quasiperiodic analysis similar to the one of crystal surfaces in terms of particular netplanes. To obtain exact results we cannot rely on features seen in a model patch. Instead we make full use of the technique of windows or coding for quasiperiodic structures. The general principles of the window technique have been described in several monographs on quasicrystals. We illustrate them on the well-known one-dimensional Fibonacci paradigm. We then apply the unique lifting and projection method between the physical (parallel) and the window (perpendicular) space, called the starmap by Moody [14, to the icosahedral F-module, to the tiling, and to its decoration. It turns out that our main results can be expressed in terms of the one-dimensional Fibonacci system. 
We now survey the model input and the content of the following sections. Our bare tiling model has the following data: We adopt the $\mathcal{T}^{*(2 F)}$ tiling model projected from the face-centered hypercubic lattice $(2 \mathrm{~F}) \sim D_{6}$ in $E^{6}$. Upon scaling by a factor 2 , the lattice $(2 \mathrm{~F})$ comprises the even vertex points (even index sum) of the full hypercubic lattice $P$ whose projection was given in [9]. For a full description of the tiling and its projection we refer to [10]. Its vertex points are projected lattice points. We shall use two units of length: (5) is the length along 5fold axes of the six basis vectors $e_{i}, i=1, \ldots, 6$ of the hypercubic lattice, projected to the two invariant icosahedral subspaces $E_{\|}$or $E_{\perp}$ respectively. Along projected 2fold axes we choose the standard length (2) $=\frac{2}{\sqrt{\tau+2}}$ (5). To convert to atomic distances in i-AlPdMn we adopt from [4, 11] the $\tau$-scaled short edge length of the tiling,

$$
s=\tau(2)=\frac{2 \tau}{\sqrt{\tau+2}}(5),(5) \rightarrow 4.56 \AA .
$$

The window of the vertex points for the tiling is in $E_{\perp}$ the triacontahedron [8], [10] shown in Fig.5. The tiling is decorated according to Elser [4] with dodecahedral Bergman clusters [11]. The midpoints of these Bergman clusters are placed on the projected odd vertex points of the hypercubic lattice. Their edge length is $\tau^{-1}(2)=2.96 \AA$, their height along a 5fold direction is $\frac{2 \tau^{2}}{\tau+2}(5)=6.60 \AA$. For all other atomic positions, most of which do not enter the present analysis, we refer to [1, 11].

In section 2 we develop the window technique for the bulk tiling and its planes perpendicular to 5fold axes. We start in 2.1 with the Fibonacci tiling and explain the technique of windows. We briefly describe the icosahedral tiling $\mathcal{T}^{*(2 F)}$ for the F-phase, section 2.2, and planes of vertex points perpendicular to a 5 fold axis in a 3D space $E_{\|}$, section 2.3, and give their windows in $E_{\perp}$. We shift between these planes along Fibonacci lines, section 2.4, and show that most of the vertex points belong to a system of shifted planes, section 2.5. In section 2.6 we interpret the terraces found in the STM experiment as terminations of the bulk model. From the tiling model we prove the existence of a full Fibonacci sequence of planes and of a spacing as found in the STM experiment, and we predict variations of the density of vertex points along the sequence, with bounds from the observed Fibonacci string.

In section 3 we use the decoration of the tiling to infer more structure information within the planes from other atomic positions of the tiling model. In particular we look for pentagonal structures as seen in the STM experiments [15]. We consider the dodecahedral Bergman clusters of the Elser model [4] on the tiling. The dodecahedra have two pentagonal vertex sets of the same orientation perpendicular to a 5fold axis. The corresponding cutting planes 
are transformed in subsection 3.1 by lifting and projection to the perpendicular space. Their window description with respect to the triacontahedron is derived. The correlation with vertex points of the tiling gives rise to three alternative models for the structure within planes. The predicted density of vertex points and pentagons is derived in exact form in section 3.2. In subsection 3.3 we compute in closed form the Patterson function within planes for vertex points and pentagon centers.

The bulk structure of the tiling model, analyzed here up to the level of Bergman clusters, displays for the planes a repetition and structure pattern in line with the terrace structure found in the experiments [15] which stimulated the present analysis. A complementary approach to the terrace structure, based on generating a model patch, is given in [12] and confirms the present analysis.

\section{Tilings and windows.}

\subsection{Fibonacci lines, their windows, and search for the string LLSLLSLSLL.}

We recall the well-known projection and window technique for the Fibonacci tiling $\mathcal{T}$. We shall emphasize the window technique since it will be needed when we apply in sections 2.4-2.6 Fibonacci lines to the icosahedral tiling. Let $\Lambda$ be the square lattice in $2 \mathrm{D}$ whose edge length we adjust for convenience to $\sqrt{\tau+2}, \tau=\frac{1}{2}(1+\sqrt{5})$. In a lattice basis the points of $\Lambda$ are

$$
x=n_{1} e_{1}+n_{2} e_{2} .
$$

In a system of coordinates $\left(x_{\|}, x_{\perp}\right)$ rotated by $\phi: c=\cos (\phi)=\frac{\tau}{\sqrt{\tau+2}}, s=$ $\sin (\phi)=\frac{1}{\sqrt{\tau+2}}$ wrt. the natural basis, the basis vectors are

$$
e_{1}=(c,-s) \sqrt{\tau+2}, e_{2}=(s, c) \sqrt{\tau+2},
$$

and the coordinates of the lattice points become

$$
\begin{aligned}
\left(x_{\|}, x_{\perp}\right) & =\left(n_{1} e_{1 \|}+n_{2} e_{2 \|}, n_{1} e_{1 \perp}+n_{2} e_{2 \perp}\right) \\
& =\left(x_{\|}\left(n_{1}, n_{2}\right), x_{\perp}\left(n_{1}, n_{2}\right)\right)=\left(n_{1} \tau+n_{2},-n_{1}+n_{2} \tau\right) .
\end{aligned}
$$

The projections $\left(x_{\|}, x_{\perp}\right)$ form two $\tau$-modules on orthogonal lines $E_{\|}, E_{\perp}$ respectively. There is a unique map $x_{\|}\left(n_{1}, n_{2}\right) \Leftrightarrow x_{\perp}\left(n_{1}, n_{2}\right)$ between these modules, corresponding to the star map of Moody [14], and there is a unique lifting of $x_{\|}\left(n_{1}, n_{2}\right)$ or $x_{\perp}\left(n_{1}, n_{2}\right)$ into a point of $\Lambda$. The projections $x_{\|}(\Lambda), x_{\perp}(\Lambda)$ cover $E_{\|}, E_{\perp}$ dense and uniformly. 
Upon choosing in $E_{\perp}$ the window $f_{\perp}:=(-1, \tau]$, whose length $\left|w_{\perp}\right|=\tau+1$ is the projection of a unit square to $E_{\perp}$, the vertex set of the Fibonacci tiling $\mathcal{T}$ in $E_{\|}$is defined as

$$
v(\mathcal{T})=\left\{x_{\|}\left(n_{1}, n_{2}\right) \mid x_{\perp}\left(n_{1}, n_{2}\right) \in f_{\perp}\right\} .
$$

The endpoints of the window are restricted in order to avoid ambiguities. When $\mathcal{T}$ is lifted back into $\Lambda \in E^{2}$, it forms the vertex set of a continuous staircase formed by edge lines as shown in Fig.1.
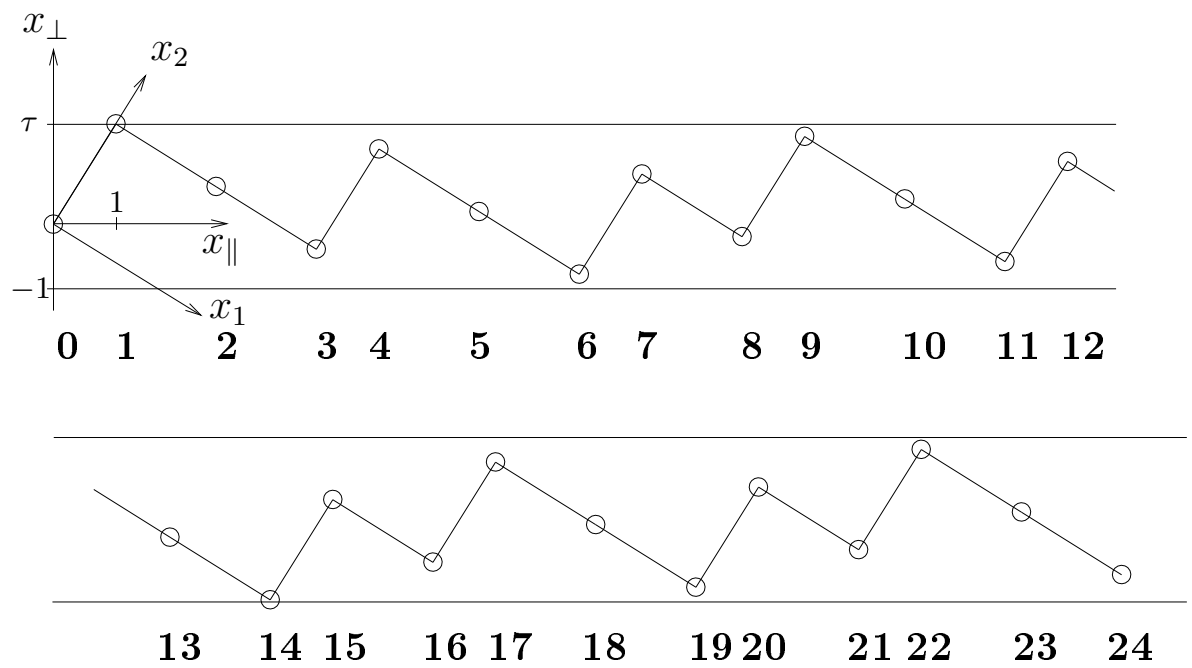

Fig.1 The Fibonacci tiling is the projection of a staircase, formed by edge lines in a square lattice, to a line $E_{\|}$of slope $\tau^{-1}$. The successive vertices $x_{\|}$of the staircase may be enumerated by the single integer $N=n_{1}+n_{2}$. Projected to $x_{\|}$, the edge lines form the Fibonacci tiling with two tiles S,L of length 1, $\tau$ respectively. The projections $x_{\perp}(N)$ of the vertices to the orthogonal space $E_{\perp}$ fall into a window $f_{\perp}=(-1, \tau]$ of length $\tau+1$.

The projections of the steps to $E_{\|}$form the familiar Fibonacci tiling with two tiles $\mathrm{S}, \mathrm{T}$ of length $1, \tau$ respectively. With $x_{\|}$increasing, adjacent tiles form the vertex configurations LS, LL, or LS respectively. The windows in $E_{\perp}$ for these vertex configurations can be shown to form subwindows of $f_{\perp}$ given by

$$
\begin{aligned}
f_{\perp}^{L S} & =(-1,0], \\
f_{\perp}^{L L} & =(0, \tau-1], \\
f_{\perp}^{S L} & =(\tau-1, \tau] .
\end{aligned}
$$

We now wish to compare and analyze Fibonacci tilings with different starting points. Because of the uniform dense covering, we may choose in $E_{\perp}$ an 
arbitrary initial point $c_{\perp} \in w_{\perp}$ and associate to it an initial point of a tiling $\mathcal{T}\left(c_{\perp}\right)$. We label the initial vertex by $(0,0) \Rightarrow 0$ and the successive vertices of $\mathcal{T}\left(c_{\perp}\right)$ by the single integer $N=n_{1}+n_{2}$. From the window condition we can generate $x_{\|}(N), x_{\perp}(N)$ step by step according to

$$
\begin{aligned}
x_{\perp}(N+1)= & {\left[\begin{array}{l}
x_{\perp}(N)-1 \leftrightarrow\left(x_{\perp}(N)-1\right) \in f_{\perp}, \\
x_{\perp}(N)+\tau \leftrightarrow\left(x_{\perp}(N)+\tau\right) \in f_{\perp} .
\end{array}\right], } \\
x_{\|}(N+1)= & {\left[\begin{array}{l}
x_{\|}(N)+\tau, \\
x_{\|}(N)+1 .
\end{array}\right] }
\end{aligned}
$$

The steps in $E_{\|}$propagate the tiling by a new tile $\mathrm{L}$ or $\mathrm{S}$ respectively. For later purposes, like the determination of densities of points in section 3.2, we emphasize the propagation as a function of $\mathrm{N}$ in terms of the perpendicular coordinate in the window.

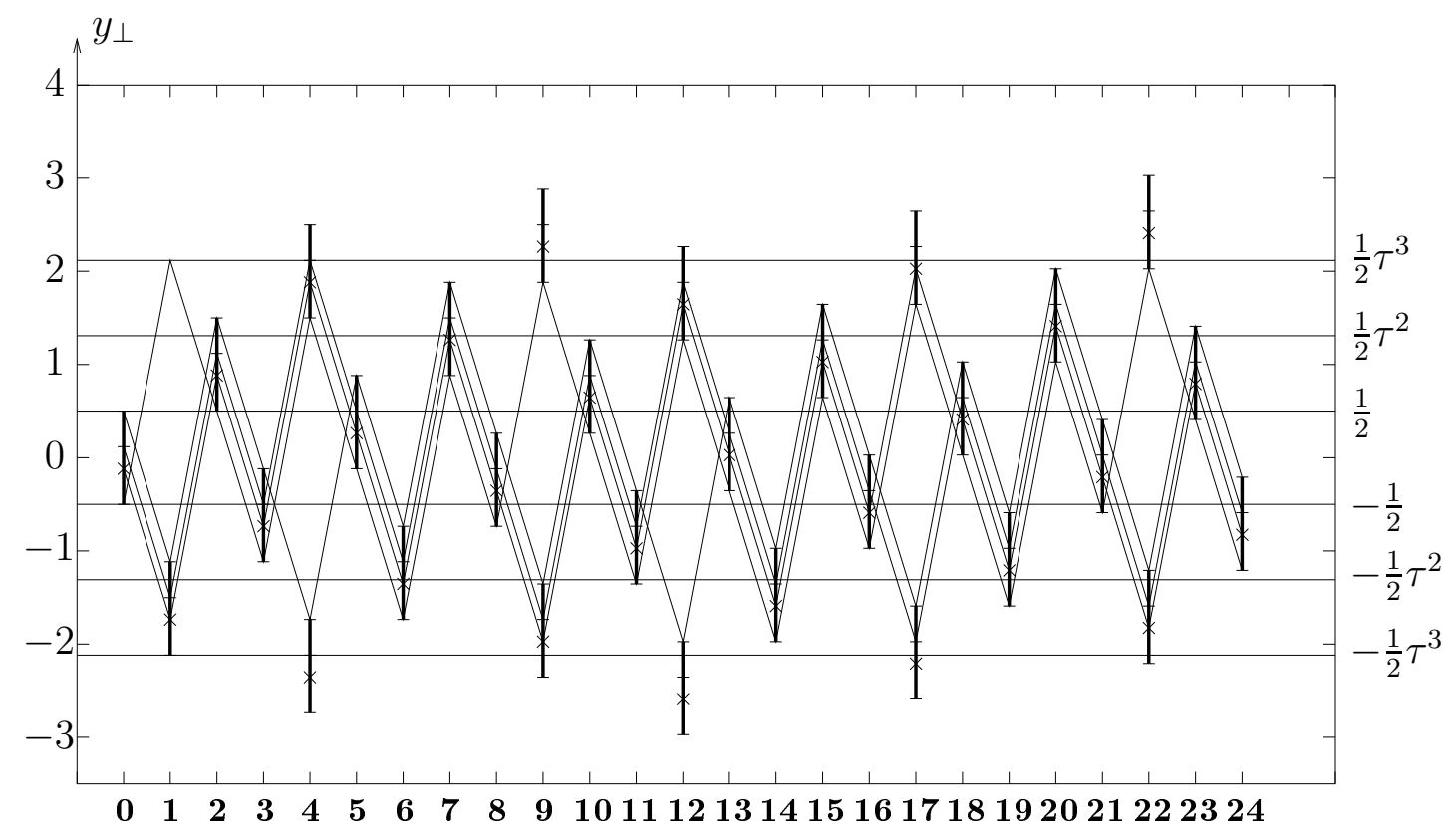

Fig.2 Four Fibonacci lines starting at an $L L$ vertex are coded by four initial points in a vertical subwindow scaled by $\tau^{-3}$. The vertical coordinate is $y_{\perp}(N)$ of eq. (8). For steps numbered from 0 to 24 , the lines connect the images in the window for these four points. Each step produces in $E_{\|}$a long or short interval of the corresponding Fibonacci line.

We adjust the perpendicular coordinate to the midpoint of the window and scale it by a factor $\tau$ to obtain the new variable

$$
y_{\perp}(N):=\tau x_{\perp}(N)-\frac{1}{2}
$$


whose window $w_{\perp}=\left(-\frac{1}{2} \tau^{3}, \frac{1}{2} \tau^{3}\right]$ now has the length $\left|w_{\perp}\right|=\tau^{3}$ with the central subwindow for LL vertex configurations of length $\left|w_{\perp}^{L L}\right|=1$. It can be shown that the subset of LL vertices of the original tiling form another Fibonacci vertex set scaled by a factor $\tau^{3}$. The function $y_{\perp}(N)$ is plotted in Fig. 2 for four initial values from the subwindow $w_{\perp}^{L L}$. This Figure illustrates the variety of sequences as a function of the initial value. Successive values are connected by straight lines. The reason for starting at an LL subwindow will become apparent when we go to the icosahedral tiling in section 2.4 .

With the window technique we search for the finite string LLSLLSLSLL found in the terrace spacing of the experiment [15]. For a Fibonacci line coded by the initial point $y_{\perp}(0)=-\frac{1}{2}$, this string occurs at the points $\mathrm{N}=9$ ... 19, compare Figs. 2,6. For other initial points, the string would occur at some other step. We infer all possible occurrences of the string as conditions with respect to the window: The string will be stable under vertical shifts $\Delta y_{\perp}(0)$ of the initial point as long as its highest value $y_{\perp}(17)$ and its lowest value $y_{\perp}(14)$ do not pass the limits $\pm \frac{1}{2} \tau^{3}$ respectively of the window $w_{\perp}$. These window conditions are independent of the initial point. Clearly the appearance of the string puts narrow bounds on the corresponding values of $y_{\perp}$, compare section 2.6 .

\subsection{Icosahedral tilings.}

The construction of 3D tilings follows the paradigm given by the Fibonacci line. The projections are now determined by requiring non-crystallographic and in particular icosahedral point symmetry after projection. It is wellknown that an icosahedral tiling $\mathcal{T}^{P}$ with two rhombohedral tiles arises by icosahedral projection to $3 \mathrm{D}$ from the primitive hypercubic P-lattice and module in $6 \mathrm{D}$ [9].

In two orthogonal $3 \mathrm{D}$ spaces $E_{\|}, E_{\perp}$ we find the six 5 fold, ten 3 fold and fifteen 2 fold axes associated with the icosahedral group. The six primitive basis vector $e_{1}, \ldots e_{6}$ of the hypercubic lattice upon projection point along 5fold axes. Their length we denote by (5), and their directions we choose as follows [10]: In $E_{\|}$we take $\cos \left(e_{1 \|}, e_{i \|}\right)=1 / \sqrt{\tau+2}, i=2 \ldots 6$ and for $i=2 \ldots 5$ pass from $e_{i \|}$ to $e_{i+1 \|}$ by a rotation around $e_{1 \|}$ with angle $2 \pi / 5$. In $E_{\perp}$ we take $\cos \left(e_{1 \perp}, e_{i \perp}\right)=-1 / \sqrt{\tau+2}, i=2 \ldots 6$ and for $i=2 \ldots 5$ pass from $e_{i \perp}$ to $e_{i+1 \perp}$ by a rotation around $e_{1 \perp}$ with angle $4 \pi / 5$. All vectors along 2 fold axes arise from projections of $\left(e_{i} \pm e_{j}\right), i \neq j$. Their shorter length we denote by (2) $=\frac{2}{\sqrt{\tau+2}}$ (5).

The icosahedral quasicrystals i-AlFeCu and i-AlPdMn from their diffraction pattern are indexed by the hypercubic face-centered or F-lattice and -module 
rather than the primitive $\mathrm{P}$-module. The hypercubic F-lattice, scaled by a factor 2 and denoted here as $(2 \mathrm{~F})$, may be viewed as the subset of even lattice points (even index sum) from the full hypercubic lattice in 6D.

Turning attention to this lattice and module, we briefly summarize the construction of the icosahedral tiling $\mathcal{T}^{*(2 F)}$ associated with the F-lattice and given in $[10]$. In both $3 \mathrm{D}$ spaces we have $6 \mathrm{D}$ modules whose bases can be formed for example from three short and three long vectors along three selected 2fold axes. By the unique lifting and projection, there is a one-to-one map $q_{\|} \Leftrightarrow q_{\perp}$, the star map of [14], between points $q_{\|}$and $q_{\perp}$ of the two modules in $E_{\|}$and $E_{\perp}$. For simplicity we suppress the basis and the six integers in $q_{\|}, q_{\perp}$ which generalize eq.(4) and underlie this map. The vertex points of the tiling $\mathcal{T}^{*(2 F)}$ are, as a generalization of eq.(5), given by

$$
v\left(\mathcal{T}^{*(2 F)}\right)=\left\{q_{\|} \mid q_{\perp} \in \text { triacontahedron }\right\},
$$

i.e. the projections $q_{\|} \in E_{\|}$of those lattice points whose projections $q_{\perp} \in E_{\perp}$ fall into the triacontahedral window, compare Fig.5. The projections $\left\{q_{\perp}\right\}$ fill the triacontahedron dense and uniformly. The triacontahedron is the icosahedral projection to $E_{\perp}$ of the Voronoi or Wigner-Seitz cell of the Flattice in 6D. The tiling $\mathcal{T}^{*(2 F)}$ has six tetrahedral tiles. In its present simple form we need only two tetrahedra with 3fold symmetry axis. The vertices of these two tetrahedra coincide with four even vertices of the two rhombohedra associated with the tiling $\mathcal{T}^{P}$. The simple form of the tiling $\mathcal{T}^{*(2 F)}$ is fully described by putting atoms into positions on the full rhombohedral tiles but allowing for the distinction of even and odd vertices, as is done in the Elser model [4, 11]. We shall need only the even and odd vertex points.

The relation of the tilings $\mathcal{T}^{*(2 F)}$ and $\mathcal{T}^{P}$ may be summarized as follows: The triacontahedral windows for the vertex sets coincide. The modules differ from one another: The $(2 \mathrm{~F})$ module is the even submodule of the $\mathrm{P}$ module. By expanding each of the two 3fold symmetric tetrahedra back into the corresponding rhombohedron and dropping the distinction between even and odd vertices we can locally derive $\mathcal{T}^{P}$ from $\mathcal{T}^{*(2 F)}$.

\subsection{Planes perpendicular to 5fold axes.}

We turn to planes in the tiling $\mathcal{T}^{*(2 F)}$. Fix in $E_{\|}$a 5 fold axis parallel to $e_{1 \|}$ as in Fig. 4 and consider vertex points $q_{\|}$in a plane perpendicular to it. Next we pass to $E_{\perp}$, consider the corresponding 5 fold axis parallel to $e_{1 \perp}$ and the images $q_{\perp}$ of the vertex points $q_{\|}$from the plane under the one-to-one map. It turns out that these images $q_{\perp} \in E_{\perp}$ lie again in a plane perpendicular to the 5 fold axis. In addition they must be points from the triacontahedron. Hence we get the result: 

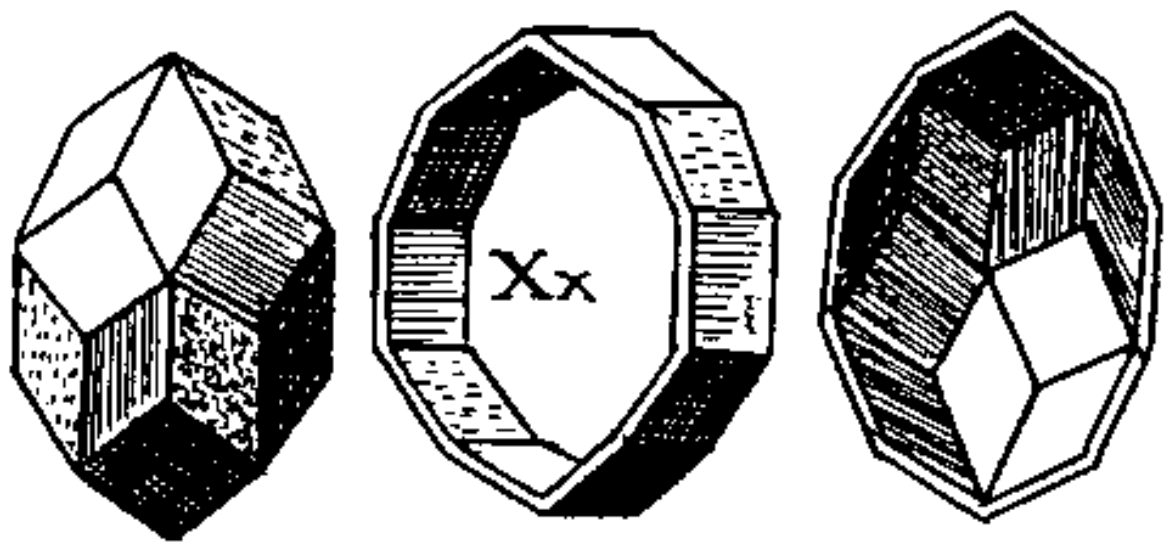

Fig.3 Kepler's decomposition of the triacontahedron into a central decagonal prism $X x$ and two shells.

Prop 1: The window for vertex points $q_{\|}$from the tiling $\mathcal{T}^{*(2 F)}$ in a fixed plane perpendicular to a 5 fold axis is in $E_{\perp}$ the intersection of a plane perpendicular to the corresponding 5 fold axis with the triacontahedron.

The triacontahedron is shown in Fig. 5 in a view perpendicular to a 5 fold axis. The distance from the center to a 5 fold vertex is $\tau$ (5), where (5) is the standard length along a 5fold axis. The triacontahedron with respect to this 5 fold axis has a central decagonal prism of thickness $\frac{2 \tau^{-1}}{\tau+2} \tau$ (5). J. Kepler in 1619 [8 not only introduced the triacontahedron, but also visualized these decagonal prisms and denoted them by the letters $X x$, see Fig.3.

The central decagonal prism of the triacontahedron when seen as a subwindow for part of the tiling $\mathcal{T}^{*(2 F)}$ has a particular siginificance, as is shown in |13]: Any planar decagonal intersection of the triacontahedron in $E_{\perp}$ determines in $E_{\|}$an infinite planar tiling TTT by two golden triangles, compare [1]. With respect to the full $3 \mathrm{D}$ tiling $\mathcal{T}^{*(2 F)}$, this planar subtiling is formed by faces of tetrahedral tiles.

The planar intersections of the triacontahedron outside the decagonal prism are windows for planes of vertex points which in general do not form a planar tiling. From the uniform covering of the window it follows that the density of vertex points in all planes is proportional to the area of its window, i.e. of the corresponding intersection of the triacontahedron. We shall compute this density in subsection 3.2 . 


\subsection{Fibonacci shifts between parallel planes.}

The planar TTT subtiling has the property that through any vertex point there passes at least one infinite Fibonacci line. In terms of its decagonal subwindow this results from the geometric property that any interior point belongs to at least one subwindow for an infinite Fibonacci line. In $E_{\|}$this Fibonacci line points along a 2fold axis associated with two vectors whose length scales by $\tau$. All subwindows for a fixed Fibonacci line are sections of length $\tau^{2}$ (2) on parallel lines perpendicular to and bounded by opposite rectangular faces of a decagonal prism.

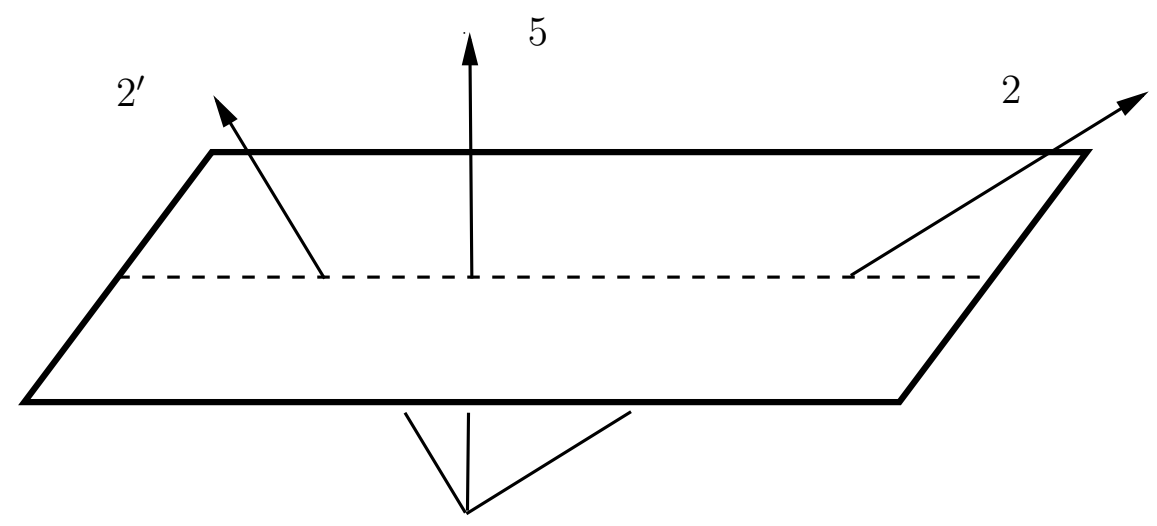

Fig. 4 Two typical perpendicular 2fold axes $2,2^{\prime}$ can be chosen within a plane with a 5fold axis 5. A plane perpendicular to this axis can be shifted from the origin by vectors along these 2 fold axes.

As the initial plane we shall choose a reference plane whose vertex points form a triangle TTT pattern. All these planes have the same highest density of vertex points, see subsection 3.2. To shift between planes perpendicular to a fixed 5fold axis, we shall use vectors along 2 fold axes outside this plane. From the orbit in $E_{\|}$of 2 fold axis with respect to the 5 fold one we pick two perpendicular 2 fold coplanar axes $2,2^{\prime}$ which form with the 5axis the angles $\arccos \left(\frac{1}{\sqrt{\tau+2}}\right)=58.3$ degrees, $\arccos \left(\frac{\tau}{\sqrt{\tau+2}}\right)=31.7$ degrees, see Fig. 4 . In the notation of [10], we choose the axis 5 along $e_{1 \|}$, the axis 2 along the short and long vectors $-\left(e_{2}+e_{3}\right)_{\|},\left(e_{1}+e_{5}\right)_{\|}$, and the axis $2^{\prime}$ along the short and long vectors $\left(e_{1}+e_{5}\right)_{\|},-\left(e_{4}+e_{6}\right)_{\|}$respectively.

The vectors along the 2 fold axis 2 from eq.(1) have the $\tau$-scaled model length $\tau$ (2), $\tau^{2}$ (2). By multiplication with the cosine of the corresponding angle we get the parallel spacings of planes perpendicular to the 5fold axis 5. These spacings become $\left(\frac{2 \tau}{\tau+2}\right.$ (5) and $\left(\frac{2 \tau^{2}}{\tau+2}\right.$ (5) respectively. By comparison, the two vectors along the 2 fold axis $2^{\prime}$ yield along the 5 fold axis spacings scaled by a factor $\tau$. Therefore the latter vectors will not generate additional parallel 
planes. We obtain the short and long spacings 4.08 and $6.60 \AA$ respectively, fully in line with the terrace spacing observed in [15] and quoted in section 1. So we have identified in the bulk tiling model the shift vectors which generate the terrace structure.

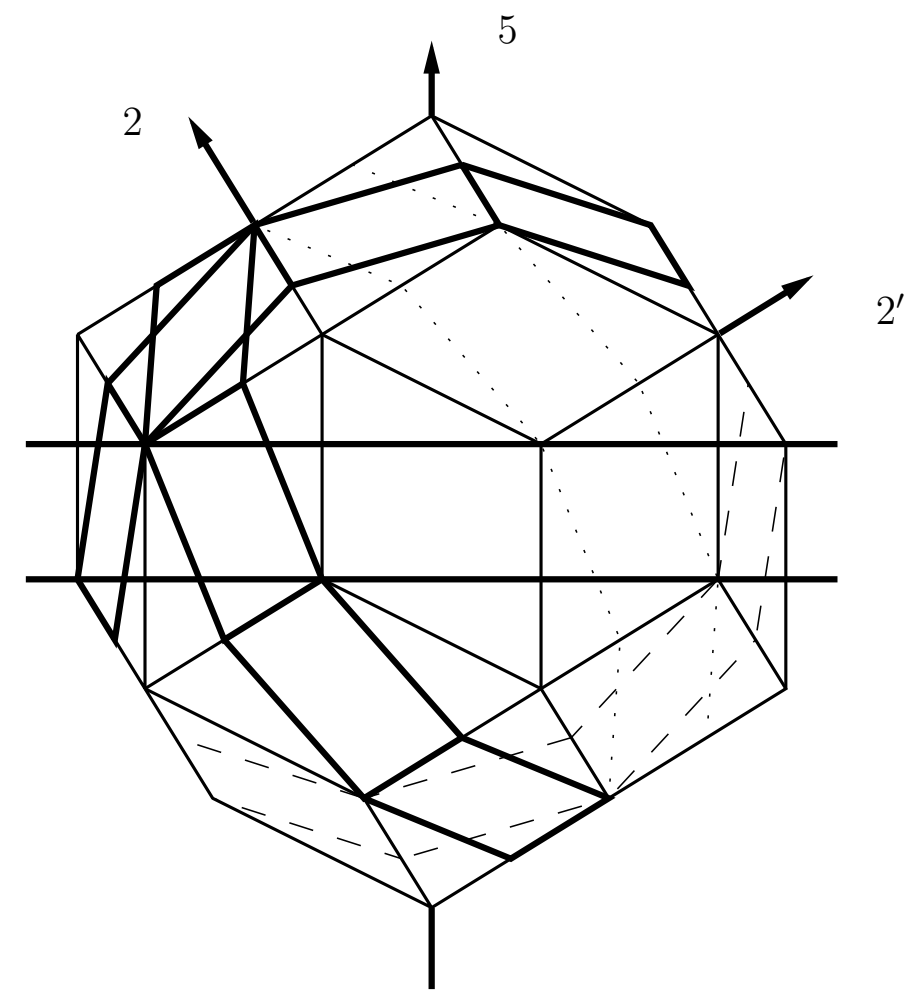

Fig. 5 Three decagonal prisms of the triacontahedron in $E_{\perp}$ : The first one has its 5 fold axis 5 in the vertical direction. The second and third prism have opposite rectangular faces perpendicular to two 2fold axes 2,2' coplanar with the axis 5 .

To assure that, starting from a fixed plane, we generate by shifts an infinite system of parallel planes, we turn to $E_{\perp}$. In $E_{\perp}$ the three axes $2,2^{\prime}, 5$ remain coplanar but the directions and angles of 2 and $2^{\prime}$ are interchanged, see Fig. 5 . We get infinite Fibonacci lines along the axes 2,2' if the vectors along these axes can be associated to decagonal prisms of the triacontahedron. Two decagonal prisms with this property and associated with 2,2' respectively are shown in Fig.5.

The initial reference plane was chosen with the triangle tiling and hence has as its window a decagonal section through the triacontahedron perpendicular to the 5 fold axis. An infinite system of parallel planes will arise, provided that we select a starting point which also belongs to a Fibonacci window associated 
with vectors say along the axis 2 . The projection of the full Fibonacci window along the 5fold axis in $E_{\perp}$ by multiplication with $\cos (2,5)=\frac{1}{\sqrt{\tau+2}}$ becomes $\frac{2 \tau^{3}}{\tau+2}$ (5). The projection of its central subwindow for LL vertices equals the thickness $\frac{2}{\tau+2}$ (5) of the decagonal prism. We conclude that, among the parallel planes shifted along the infinite Fibonacci line in the direction 2, the reference plane and in fact any dense plane occurs at the LL vertices.

We summarize the information obtained so far on parallel planes of vertex points $q_{\|}$in the tiling $\mathcal{T}^{*(2 F)}$, generated by Fibonacci lines along 2fold axes: Starting from a dense reference plane, we generate an infinite set of parallel planes. They follow a Fibonacci spacing with perpendicular short and long distances $\frac{2 \tau}{\tau+2}(5)=4.08 \AA$ and $\frac{2 \tau^{2}}{\tau+2}(5)=6.60 \AA$, fully in line with the STM observations of terraces. The dense planes occur at all LL vertices of the generating Fibonacci line. Other parallel planes in the set will have a lower density of vertex points. The string LLSLLSLSLL analyzed in section 2.1 can now be converted into a sequence of parallel planes of varying density. To complete the analysis of parallel planes, we must find out what fraction of all vertex points $q_{\|} \in \mathcal{T}^{*(2 F)}$ is reached within this infinite sequence of planes.

\subsection{Parallel planes are connected by Fibonacci lines.}

We wish to show that indeed we can reach from a fixed dense reference plane most vertex points $q_{\|}$by shifts along Fibonacci lines. For this purpose we consider only those vertex points $q_{\|}$which lie on at least one infinite Fibonacci line. From the window side we know that this is the case if $q_{\perp}$ is a point from any decagonal prism. There are six such prisms, and this motivates the definition of a new window:

Def 2: Decagonal prism $X x$ approximation: We analyse only those points $q_{\perp}$ of the triacontahedral window which belong to at least one decagonal prism, that is to the union $\cup_{j}^{6}$ prism $_{j}$. We omit in this approximation the points $q_{\perp}$ from small parts of the triacontahedron close to its 5fold vertices, compare Fig.5.

The vertex points $q_{\|}$, with $q_{\perp}$ belonging to this new window, have the following properties:

Prop 3: Consider vertex points $q_{\|}$in a plane parallel to a fixed dense TTT infinite reference plane. Among them there is a point on an infinite Fibonacci line which intersects (as a continuous line) the reference plane.

Proof: Through any point coded in the decagonal prism approximation there runs at least one infinite Fibonacci line. If it intersects the reference plane we are through. If it runs parallel to the reference plane, we can (proof omit- 
ted) in at most two parallel steps pass to another point with an intersecting Fibonacci line.

Prop 4: If an infinite Fibonacci line intersects as a continous line a TTT plane, it hits this plane in a vertex point.

Proof: The points of the infinite reference plane form the vertices of the planar TTT subtiling by faces, the points of the non-parallel infinite Fibonacci line form the vertices of a linear subtiling by edges of the $3 D$ tiling $\mathcal{T}^{*(2 F)}$. Both subtilings are parts, hence their intersection is a vertex of the full tiling.

The two propositions allow us to code in $E_{\perp}$ planes of vertex points, parallel in $E_{\|}$to a first dense reference plane perpendicular to 5 , by their intersections with Fibonacci lines along the axis 2 of Fig.4:

Prop 5: Any vertex plane perpendicular to a 5fold axis has at least one point connected to the reference plane by an infinite Fibonacci line. Conversely, by following all non-parallel Fibonacci lines from the reference plane we reach any parallel vertex plane.

We have shown that all vertex points $q_{\|}$such that $q_{\perp}$ belongs to the Kepler $\mathrm{Xx}$ model appear in an infinite sequence of parallel planes in the order and spacing of a Fibonacci line. The analysis of the 1D Fibonacci system given in subsection 2.1 now applies to the 3D tiling. The stepwise generation in $E_{\perp}$ of the 1D Fibonacci system shown in Fig. 2 can now be converted into a stepwise generation in $E_{\perp}$ of parallel planes, enumerated by the integer $\mathrm{N}$ : Starting at an LL vertex means starting at a dense plane. The value of the perpendicular coordinate $y_{\perp}$ yields the value of a coordinate $\eta \tau$ (5), $-1 \leq \eta \leq 1$ from the center of the triacontahedron along the 5fold axis. The explicit relation is

$$
\eta(N)=\frac{2 \tau^{-1}}{\tau+2} y_{\perp}(N) .
$$

In Fig. 6 we plot the projection of the triacontahedron together with the values of $y_{\perp}(N)$ connected by lines. Each value determines the height of a corresponding horizontal section of the triacontahedron. Since the point density in the corresponding plane of the tiling is proportional to the area of this section, Fig.6 provides insight into the variation of this density from step to step.

Note that within the Fibonacci window we do not reach the highest absolute values of $\eta$. For the additional points in Fig.6 see section 2.6. 


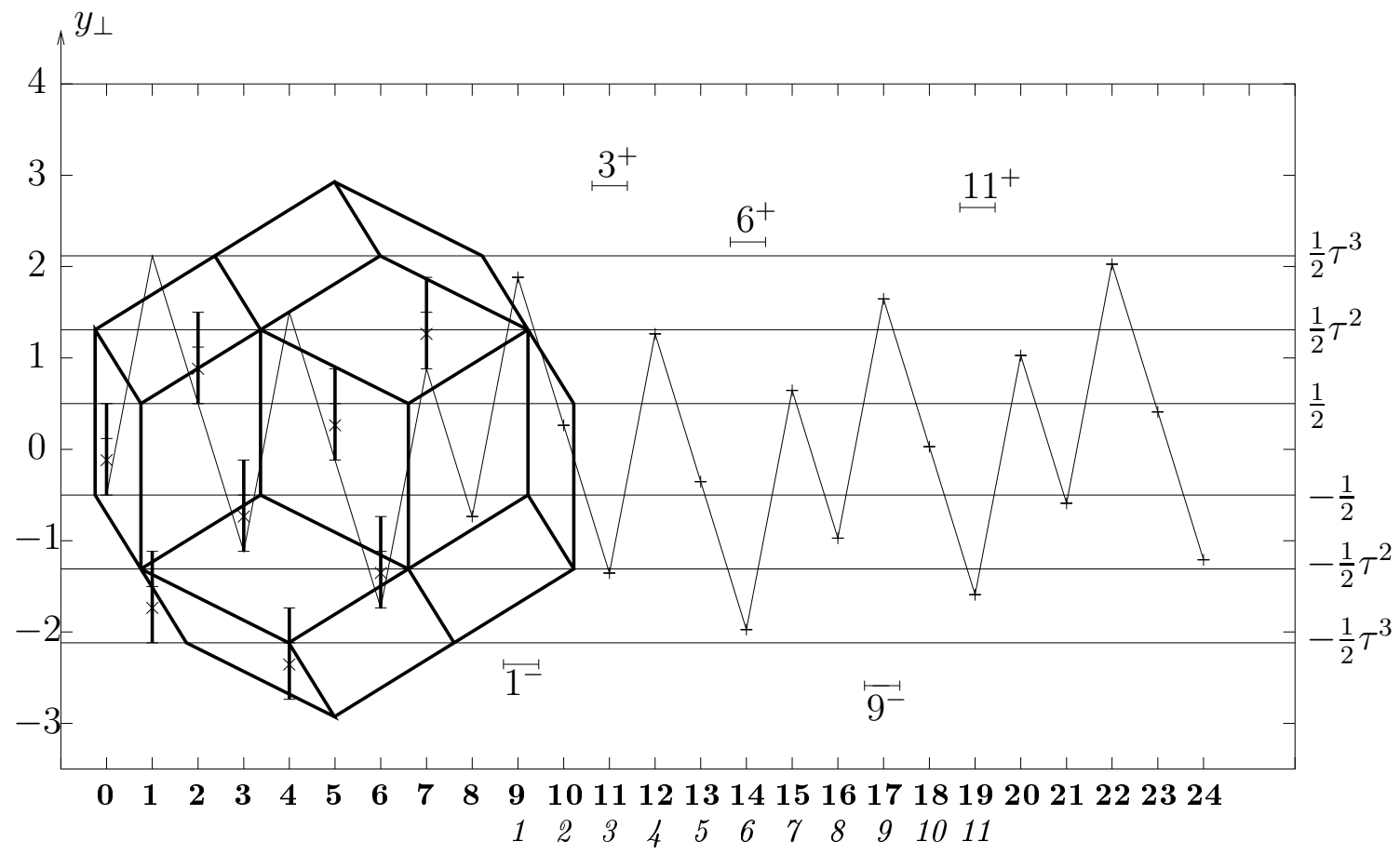

Fig.6 Vertical values of $y_{\perp}(N) \sim \eta(N)$ eq.(10) determine horizontal sections in $E_{\perp}$ of the triacontahedron as windows for planes. The values are connected by lines and follow the Fibonacci coding. The total width of the vertical window is $\frac{2 \tau^{2}}{\tau+2} \tau$ (5). The triacontahedron has the vertical diameter $2 \tau$ (5). Numbers in the second row assign planes corresponding to the terraces found in [15]. The bars $1^{-}, 9^{-}, 3^{+}, 6^{+}, 11^{+}$mark values of $\eta$ for additional lowdensity planes of vertex points.

\subsection{From planes to terraces at the surface.}

We have found in section 2.4 from the bulk model sequences of planes with a spacing that agrees with the terrace spacing found in [15]. We now interpret terraces at the surface as particular planar terminations from the bulk tiling model. From Fig.2 we have already identified a string in correspondence to the observed string of terraces. With the numbers $1 \ldots 11$ in the second horizontal row of Fig. 6 we now assign planes and values $\eta_{1}$ to eleven planes which correspond to the spacing of eleven high or low terraces found in [15]. The numbers follow the terraces in a direction into the bulk material.

The numbers $\eta_{1}$ given in Table 1 are not unique, but the appearance of the finite string puts narrow bounds on their range: Maximal shifts upwards by $\Delta \eta=\frac{2 \tau^{-1}}{\tau+2}(2 \tau-3)$ from $N=9$ or downwards by $\Delta \eta=\frac{2 \tau^{-1}}{\tau+2}(-3 \tau+5)$ from 
$N=14$ are compatible with the appearance of the string, but of course give different values of the density and of the Patterson function.

As in the patch analysis given in [12, there appear additional vertex planes in $E_{\|}$with a spacing scaled by $\tau^{-1}$ which do not yield terraces in the experiment. This narrow spacing cannot be coded by a single Fibonacci line. With respect to the triacontahedron in Fig.5, it requires a vertical shift $\Delta \eta=\frac{2 \tau^{2}}{\tau+2}$ in $E_{\perp}$. This shift can be produced by the sum of two vectors of length $\tau(2)$ pointing along the axes 2 and $2^{\prime}$ in Fig. 2 respectively. The summed vector (not parallel to the 5fold axis) connects points in the triacontahedron only if the initial point obeys $\frac{\tau}{\tau+2} \leq|\eta| \leq \frac{\tau^{2}}{\tau+2}$. In the selection of points of Fig. 6 the values $\eta$ of the corresponding 5 final points are denoted by $1^{-}, 3^{+}, 6^{+}, 9^{-}, 11^{+}$. The \pm sign codes an additional vertex plane shifted in $E_{\|}$by $\frac{2 \tau^{-1}}{\tau+2}$ in units $\tau$ (5) above or below the plane with the fixed number. The positions of these planes agree with the patch analysis of $[12 \|$. All these additional planes have a very low density of vertex points.

In section 3.2 we compute the exact model density of vertex points in the planes which decreases with the absolute value of $\eta_{1}$. From Fig. 6 it can be seen that the planes $3,6,9$ in the string have the highest values of $\left|\eta_{1}\right|$ and hence the lowest density. In the experiment [15 these planes correspond to terraces of minimum measured planar size.

\section{Clusters, pentagonal faces and cuts, and cor- relations in planes.}

We proceed to an analysis of the more detailed model structure within the planes. So far we looked at planes occupied by vertex points from the tiling. The full set of atomic positions [4, 11] comprises more points in various classes. The repetition pattern and variation of the density for parallel planes found in section 2 is a general property of the tiling and applies to any set of atomic positions within planes perpendicular to a 5fold axis. If atomic positions of two different types appear within the same initial fixed plane, their repetition pattern and variation of density follows the same pattern as found for vertex points, but may propagate from different initial conditions. The relative density and the correlation of different types of atomic positions sitting within the same plane will then show systematic variations along a sequence of planes.

We consider in this section additional atomic positions from the Bergman clusters. The vertex positions of Bergman clusters on the tiling will produce points in parallel planes. This holds true in particular for top faces of 
Bergman clusters which run perpendicular to the chosen 5fold axis. They are of particular interest as candidates for the pentagons found in [15]. Upon comparing the height of $6.60 \AA$ for the Bergman clusters with the lowest terrace spacing of $4.08 \AA$ we already conclude that these clusters are cut at quasicrystal surfaces. A second larger type of pentagons arises from a top cut at the heigth of $4.08 \AA$ through five vertices of the Bergman dodecahedra. In the full model [4, 11] of $A l P d M n$ these top face pentagons have central atoms in a lowered central position. Therefore these pentagon faces would produce at their centers the observed holes in the terraces [15]. We shall examine the correlation of both types of pentagons with vertex points of the tiling.

\subsection{Three models for correlations of pentagons and ver- tex points.}

We analyze three significant types of planes perpendicular to a 5fold axis taken from the bulk model and explore the correlation within these planes.

(i) In a first model analysis the vertex points of the tiling dominate the sequence of perpendicular planes, characterized by the values $\eta_{1}$ as before. Consider the relation of the Bergman top pentagons with respect to these planes. As mentioned in the introduction, the centers of the Bergman dodecahedra take the positions of those (odd) points of the primitive $P$-lattice which are dropped when going from $\mathrm{P}$ to $(2 \mathrm{~F})$. This has the consequence that the Bergman centers can be grouped into shifted planes perpendicular to a 5 fold axis. The planar densities of Bergman clusters in these planes must follow the same rules, minima, and maxima as given for vertex positions in sections 2.4 and 3.2 .

The height of the Bergman dodecahedra is such that a 5fold vector from the plane to the center, passing through a pentagonal face, has the length and direction of a typical vector $e_{5 \|}$, compare Fig.7. With the chosen enumeration of terraces we follow them in the direction of the vector $e_{1 \|}$, in the direction of the 5 fold axis in Fig.4. Now we look for Bergman clusters with a center displaced below and a top pentagon within a fixed plane of vertex points. 


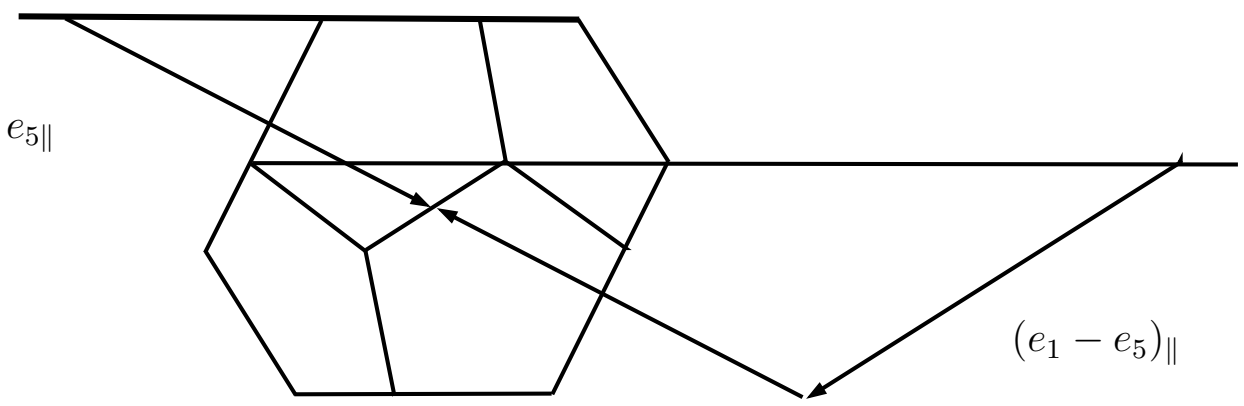

Fig.7 A Bergman dodecahedron touches with a top pentagon from below a plane of vertex points marked by the top horizontal line. The vector $e_{1 \|}$ points downwards. A typical vector downwards from the plane to the Bergman center is $e_{5 \|}$. A vector like $\left(e_{1}-e_{5}\right)_{\|}-e_{5 \|}$ runs from a planar pentagonal top cut through the dodecahedron, marked by the lower horizontal line, to the Bergman center.

Transforming the vector $e_{5 \|}$ from $E_{\|}$to $E_{\perp}$, compare section 2.2 , one finds the coding points for all centers of the Bergman dodecahedra displaced by $e_{5 \perp}$ against the direction of the axis 5 of Fig.5, hence downwards in Fig.5, from the plane coding the vertex points. So the planes with a corresponding downwards shift $\Delta \eta=\frac{1}{\tau+2}$ form another Fibonacci sequence that codes the Bergman centers. The shifted values $\eta_{2}$ for this sequence are given in the second column of Table 1 . In Fig. 8 we show the two values $\eta_{1}, \eta_{2}$ as functions of $N=0, \ldots, 24$. If the vertical shift from the crosses to the circles equals $\Delta \eta=\frac{1}{\tau+2}$, the Bergman top pentagons touch the vertex plane from below. This holds true except for the planes $N=6,11,14,19$, see Fig.8. In the Fibonacci sequence of planes of vertex points this correspond to an LS vertex, but in the planes of Bergman faces to an SL vertex. Therefore no Bergman top faces can appear in these planes of vertex points. In the string of planes $9 \ldots 19 \rightarrow 1 \ldots 11$ this would occur at the planes $11,14,19 \rightarrow 3,6,11$. These planes carry instead the larger Bergman top cut pentagons, see model (iii). Figs.8,9 demonstrate that planes formed from atomic positions of different type (vertex points or pentagon centers) follow the same Fibonacci propagation law. Due to systematic shifts in the parameter $\eta$ for different types, the corresponding densities, which are functions of this parameter, propagate differently even for atomic positions of different type within the same initial plane. 


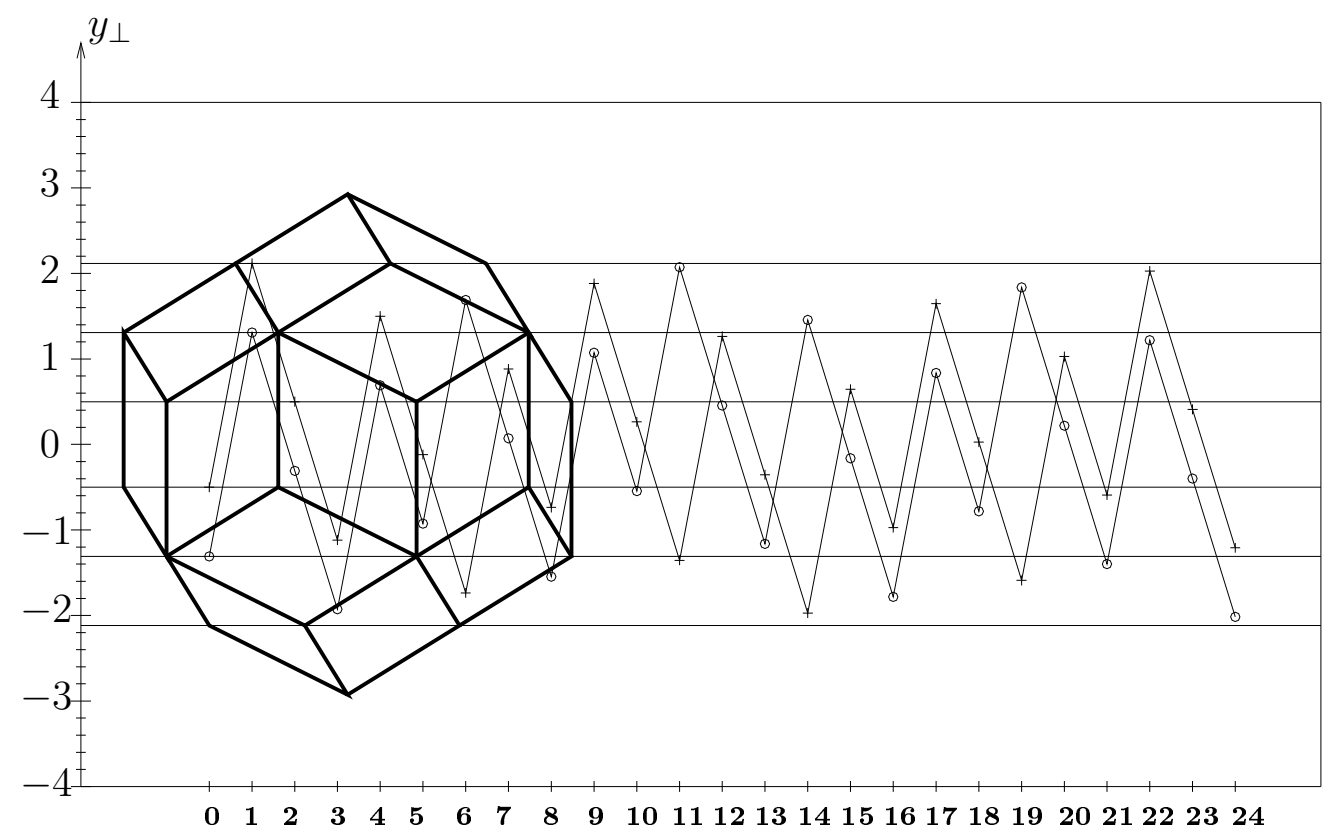

Fig.8. The values $y_{\perp}(N) \sim \eta(N)$ for $N=0, \ldots, 24$ in model (i) determine horizontal sections of the triacontahedron as windows for planes of vertex points $\left(\eta_{1}\right.$, crosses) or of centers for Bergman top pentagons $\left(\eta_{2}\right.$, circles).

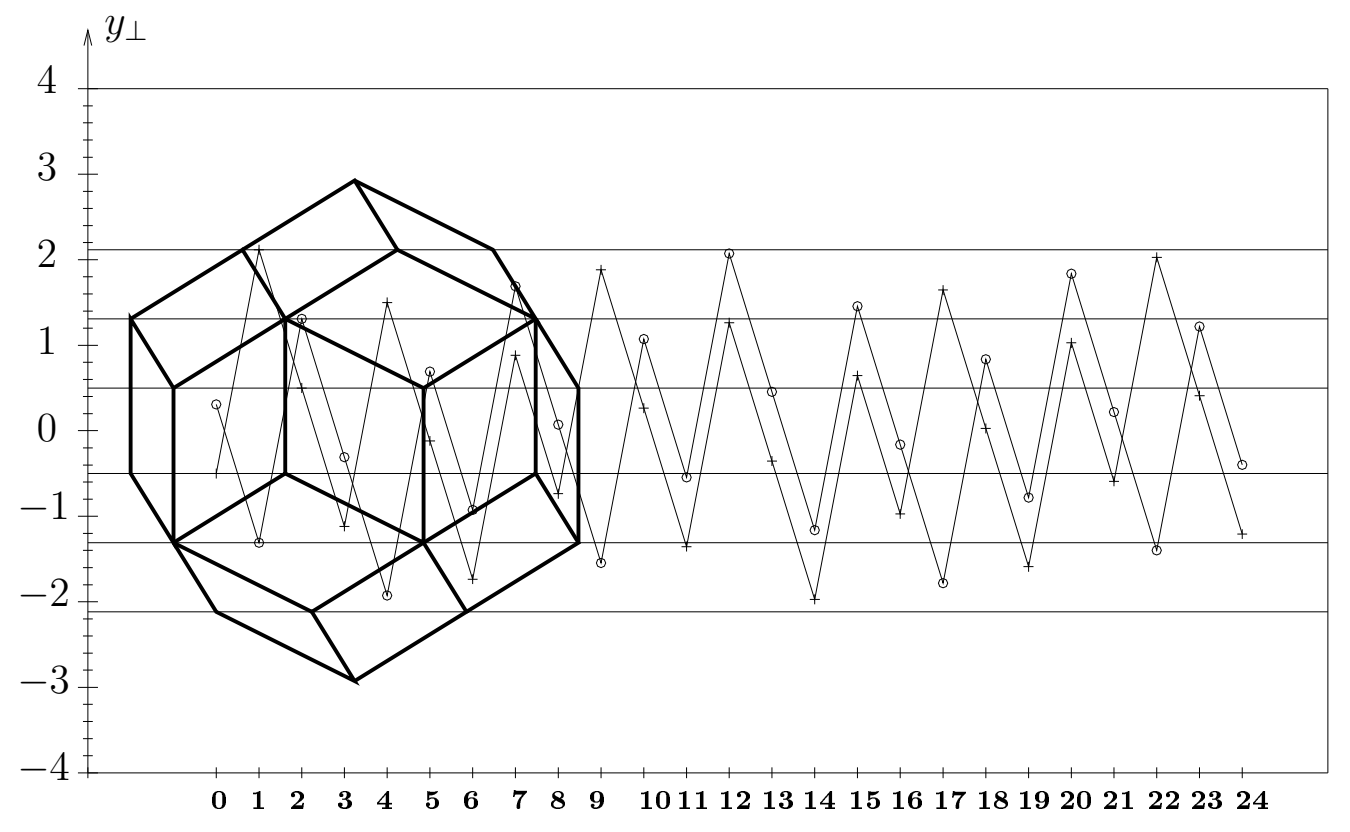

Fig.9. The values $y_{\perp}(N) \sim \eta(N)$ for $N=0, \ldots, 24$ in model (ii) determine horizontal sections of the triacontahedron as windows for planes of centers for Bergman top pentagons ( $\eta_{1}$, crosses) or of vertex points $\left(\eta_{3}\right.$, circles). 
(ii) In the second model analysis of the planes determined by $\eta_{1}$ we assume that they are dominated by top pentagons of Bergman dodecahedra located below the plane, compare Fig.9. The additional presence of vertex points on these planes is now coded by a positive shift $\Delta \eta=\frac{1}{\tau+2}$. In Table 1 we give the new values $\eta_{3}$ for these vertex planes and show them in Fig.9. Additional vertex points appear except in the selected planes $9,17 \rightarrow 1,9$.

(iii) In the third model analysis we assume that the planes determined by $\eta_{1}$ are dominated by pentagons, scaled by $\tau$ and corresponding to top cuts through Bergman dodecahedra below the plane. A typical vector from the plane for this cut to the center of the Bergman cluster in the notation of section 2.2 is $\left(-e_{5 \|}+e_{1 \|}\right)-e_{5 \|}$ indicated in Fig.7. The edge size (2) $=4.78$ $\AA$ of the pentagons would be in line with the holes observed in [15, and it would also lead to a central hole. Again we ask about the presence of additional vertex points. From the relative position of these pentagons to the vertex planes as shown in Fig.7 we deduce in $E_{\perp}$ a shift $\Delta \eta=\frac{2 \tau+1}{\tau+2}$. At $N=0$ this shift modulo the window size becomes $\frac{-1}{\tau+2}$ which then generates the values $\eta_{3}$ given in Table 1 and shown in Fig.9. Vertex points can occur only if the relative shift from crosses to circles is $\Delta \eta=\eta_{3}-\eta_{1}=\frac{2 \tau+1}{\tau+2}$. This happens only in the selected planes $9,17 \rightarrow 1,9$, otherwise there are no vertex points within these planes. A further shift analysis shows that any plane containing Bergman top pentagons cannot contain Bergman cut pentagons and vice versa. In model (iii) there appear Bergman top cut pentagons in densest planes. A closer inspection shows that these pentagons share vertices and form an almost connected graph.

The three model cases considered yield three interpretations of bulk planes as terminations for the terraces observed in [15], all in line with the relative spacing. In cases (i,ii) the planes carry vertex points or pentagons corresponding to the faces of Bergmann dodecahedra. In case (iii) the planes carry the larger cut pentagons and almost no vertex points.

\subsection{Planar density of atomic positions.}

We compute the exact area $F(\eta)$ of a planar section of the triacontahedron as a function of $\eta,-1 \leq \eta \leq 1$. This function is proportional to the exact density of vertex points $D(\eta)$ in the plane coded by this section. The result is

$$
\begin{aligned}
& 0 \leq|\eta| \leq \frac{\tau^{-1}}{\tau+2}: \\
& F(\eta)=(\tau+2)^{-3 / 2}[10 \tau],
\end{aligned}
$$




$$
\begin{aligned}
& \frac{\tau^{-1}}{\tau+2} \leq|\eta| \leq \frac{\tau}{\tau+2}:(\tau+2)^{-3 / 2}\left[10 \tau-5 \frac{(\tau+2)^{2}}{\tau}\left(|\eta|-\frac{\tau^{-1}}{\tau+2}\right)^{2}\right], \\
& F(\eta)= \\
& \frac{\tau}{\tau+2} \leq|\eta| \leq \frac{\tau^{2}}{\tau+2}: \\
& F(\eta)=(\tau+2)^{-3 / 2} \\
& \quad\left[10+5 \frac{(\tau+2)^{2}}{\tau}\left(\frac{\tau^{2}}{\tau+2}-|\eta|\right)^{2}-5(\tau+2)^{2}\left(|\eta|-\frac{\tau}{\tau+2}\right)^{2}\right], \\
& \frac{\tau^{2}}{\tau+2} \leq|\eta| \leq 1: \\
& F(\eta)=(\tau+2)^{-3 / 2}\left[5(\tau+2)^{2}(1-|\eta|)^{2}\right] .
\end{aligned}
$$

The function $F(\eta)$ is plotted in Fig.10. The maximum is $F(0)=10 \tau(\tau+$ $2)^{-3 / 2}=2.3511$, the maximum value of $\eta$ for the Fibonacci sequence of planes is

$$
|\eta|=\tau^{2}(\tau+2)^{-1}=0.7236
$$

These values are marked by vertical and horizontal lines in Fig.10.

The density can be converted into the absolute density of vertex points by considering the densest planes with a triangle pattern and its vertices: In the triangle pattern, each triangle contributes, because of the sum $\frac{1}{2} 2 \pi$ of its angles, a weight $\frac{1}{2}$ to the number of vertex points. In terms of the short edge length $s$, the area $f_{1}, f_{2}$ and relative frequency $\nu_{1}, \nu_{2}$ of the large and small triangle are

$$
f_{1}=s^{2} \frac{\tau}{4} \sqrt{\tau+2}, f_{2}=\tau^{-1} f_{1}, \nu_{2}=\tau^{-1} \nu_{1}
$$

These expressions yield for the density of vertex points (number of vertices per unit area) the value

$$
D(0)=\frac{1}{2 f_{1}} \frac{\tau^{3}}{\tau+2}=s^{-2} \frac{2 \tau^{2}}{(\tau+2)^{3 / 2}}
$$

In the present model we put for the short edge length $s=\tau(2)=\tau \frac{2}{\sqrt{\tau+2}}$ (5) and (5) $=4.56 \AA$ eq.(1) to obtain the model value

$$
D_{\max ,(i)}=D(0)=12.6 \cdot 10^{-3} \AA^{-2} .
$$

The density of vertex points in a plane for fixed $\eta$ is now computed as

$$
D(\eta)=D(0) \frac{F(\eta)}{F(0)} .
$$


The lowest density in a Fibonacci sequence of planes from eqs. $(14,15)$ is

$$
D_{\min }=\frac{1}{2 \tau} D(0)=0.3090 D(0) .
$$

These values of the density refer to vertex positions in the tiling. All other atomic positions on the tiles will of course lead to other specific atomic densities and correlations.

The maximum density of vertex points, associated with model (i), is given in eq. (16). The same maximal density applies to the centers of Bergman top faces in model (ii). Each pentagon contributes 5 vertex positions which would yield a factor 5 for the pentagon vertex density. For model (iii) with the larger pentagons, the maximum density of centers is still the same. For the density of pentagon vertices one should not multiply by a factor 5: It turns out that these larger pentagons in a dense plane can share vertices. An exact computation of the maximum density for the vertices of large pentagons yields (proof omitted)

$$
D_{\max ,(i i i)}=\frac{7 \tau+4}{\tau^{3}} D_{\max , i}=3.6180 D_{\max ,(i)} .
$$

From the density we can compute the average distance by comparison for example with a tiling by equilateral triangles. For such a tiling, the density of points is related to the edge length $t$ by

$$
D_{3}(t)=t^{-2} \frac{2}{\sqrt{3}}
$$

If we equate this density with the expression eq.(15) found in the dense vertex planes, we obtain an equivalent distance $t_{e q}=9.5 \AA$.

In columns 5-7 of Table 1 we give $F$ for the three values of $\eta$. Note that, in view of the three models discussed in subsection 3.1, the three values of $F$ in a row do not always refer to the same plane. 


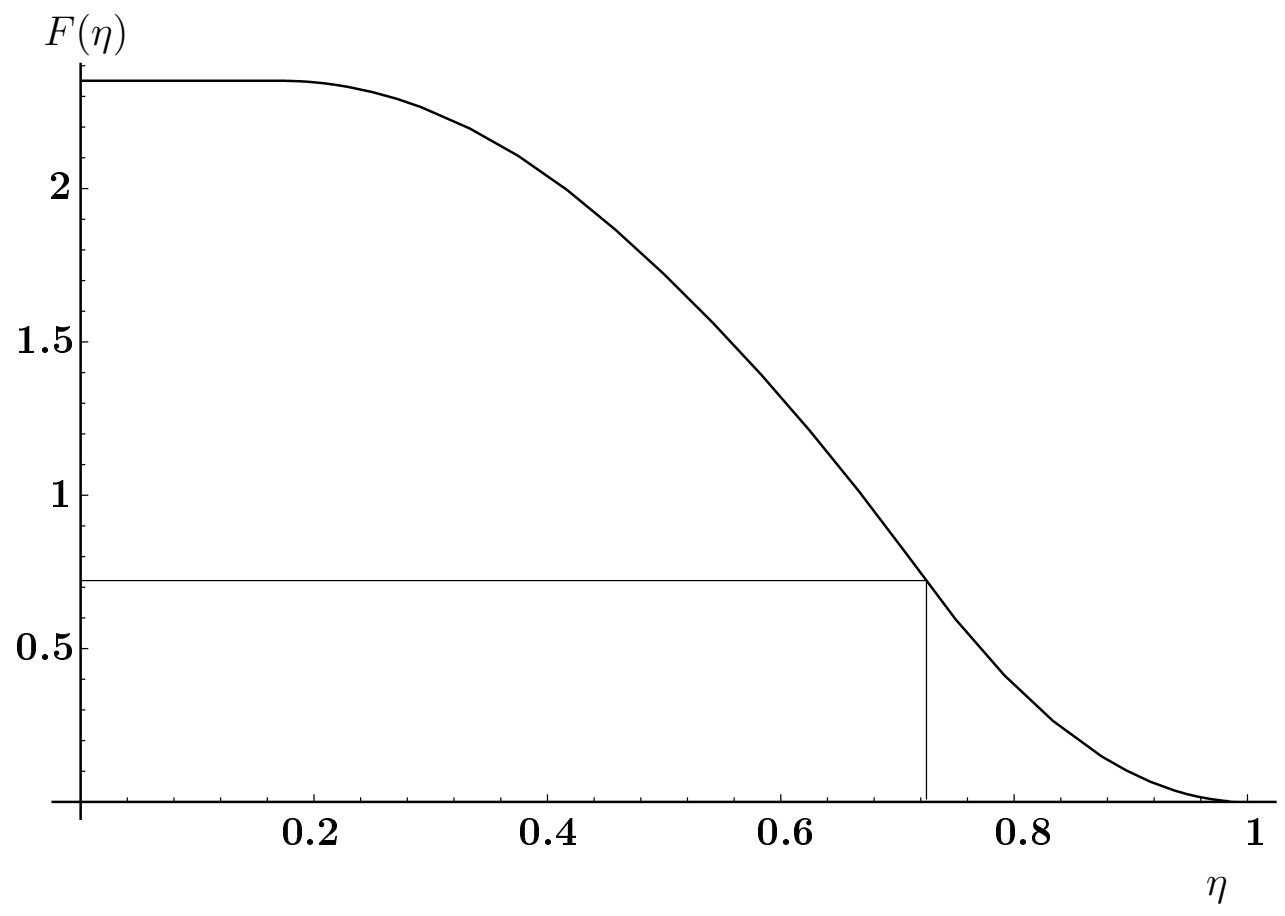

Fig.10 The function $F(|\eta|)$ for a plane fixed by $\eta \geq 0$ is in $E_{\perp}$ the area of a planar section of the triacontahedron. The horizontal line marks the lowest value of $F$, the vertical line the highest value of $|\eta|$ in a Fibonacci sequence of planes. In $E_{\|}, F(|\eta|)$ is proportional to the density of points or pentagon centers within a plane. 
Table 1: The values $\eta_{1}, \eta_{2}, \eta_{3}$ as functions of $N=0, \ldots, 24$ code inside the triacontahedron Fibonacci sequences of planes perpendicular to a 5 fold axis. These values are associated alternatively with vertex points, pentagonal faces and cuts of Bergman dodecahedra. Columns $5-7$ give the value of $F\left(\eta_{i}\right)$, equal to the area of planar sections through the triacontahedron and proportional to the relative density of points. The rows $9 \ldots 19$ are put in correspondence with the terraces $1 \ldots 11$ of [15].

\begin{tabular}{|r|r|r|r|c|c|c|}
\hline $\mathrm{N}$ & $\eta_{1}$ & $\eta_{2}$ & $\eta_{3}$ & $F\left(\eta_{1}\right)$ & $F\left(\eta_{2}\right)$ & $F\left(\eta_{3}\right)$ \\
\hline 0 & -0.1708 & -0.4472 & 0.1056 & 2.3511 & 1.9021 & 2.3511 \\
\hline 1 & 0.7236 & 0.4472 & -0.4472 & 0.7265 & 1.9021 & 1.9021 \\
\hline 2 & 0.1708 & -0.1056 & 0.4472 & 2.3511 & 2.3511 & 1.9021 \\
\hline 3 & -0.3820 & -0.6584 & -0.1056 & 2.0891 & 1.0541 & 2.3511 \\
\hline 4 & 0.5125 & 0.2361 & -0.6584 & 1.6746 & 2.3261 & 1.0541 \\
\hline 5 & -0.0403 & -0.3167 & 0.2361 & 2.3511 & 2.2260 & 2.3261 \\
\hline 6 & -0.5931 & 0.5777 & -0.3167 & 1.3507 & 1.4162 & 2.2260 \\
\hline 7 & 0.3013 & 0.0249 & 0.5777 & 2.2510 & 2.3511 & 1.4162 \\
\hline 8 & -0.2515 & -0.5279 & 0.0249 & 2.3129 & 1.6164 & 2.3511 \\
\hline 9 & 0.6430 & 0.3666 & -0.5279 & 1.1269 & 2.1259 & 1.6164 \\
\hline 10 & 0.0902 & -0.1862 & 0.3666 & 2.3511 & 2.3497 & 2.1259 \\
\hline 11 & -0.4626 & 0.7082 & -0.1862 & 1.8512 & 0.8067 & 2.3497 \\
\hline 12 & 0.4318 & 0.1554 & 0.7082 & 1.9508 & 2.3511 & 0.8067 \\
\hline 13 & -0.1210 & -0.3974 & 0.1554 & 2.3511 & 2.0495 & 2.3511 \\
\hline 14 & -0.6738 & 0.4971 & -0.3974 & 0.9796 & 1.7311 & 2.0495 \\
\hline 15 & 0.2207 & -0.0557 & 0.4971 & 2.3365 & 2.3511 & 1.7311 \\
\hline 16 & -0.3321 & -0.6085 & -0.0557 & 2.1982 & 1.2835 & 2.3511 \\
\hline 17 & 0.5623 & 0.2859 & -0.6085 & 1.4800 & 2.2733 & 1.2835 \\
\hline 18 & 0.0095 & -0.2669 & 0.2859 & 2.3511 & 2.2969 & 2.2733 \\
\hline 19 & -0.5433 & 0.6276 & -0.2669 & 1.5565 & 1.1980 & 2.2969 \\
\hline 20 & 0.3512 & 0.0748 & 0.6276 & 2.1600 & 2.3511 & 1.1980 \\
\hline 21 & -0.2016 & -0.4780 & 0.0748 & 2.3456 & 1.7986 & 2.3511 \\
\hline 22 & 0.6928 & 0.4164 & -0.4780 & 0.8851 & 1.9966 & 1.7986 \\
\hline 23 & 0.1400 & -0.1364 & 0.4164 & 2.3511 & 2.3511 & 1.9966 \\
\hline 24 & -0.4128 & -0.6892 & -0.1364 & 2.0070 & 0.9033 & 2.3511 \\
\hline
\end{tabular}

The three models (i,ii,iii) given in subsection 3.1 yield different density values $F$. In model (i), the density of vertex points and centers of Bergman top faces is given by $F\left(\eta_{1}\right)$ and, with exceptions, by $F\left(\eta_{2}\right)$. In the plane $16 \rightarrow 8$, the density of Bergman faces becomes $D=6.810^{-3} \AA^{-2}$. respectively. In model (ii), the density of Bergman top faces and vertex points is given by $F\left(\eta_{1}\right)$ 
and with exceptions by $F\left(\eta_{3}\right)$. respectively. In model (iii), the density of Bergman top cuts is again given by $F\left(\eta_{1}\right)$.

The density of pentagonal holes in the experimental data [15 has approximately $\| 12$ the value $D=4.210^{-3} \AA^{-2}$. This value would favour model (i) with Bergman top faces in planes dominated by vertex points.

\subsection{Patterson analysis in planes.}

Consider the general Patterson function $P\left(x_{\|}\right)$of a quasiperiodic point set with a window $W$ at each point of the lattice $\Lambda$. Let $v_{\|}$be a shift vector projected from a lattice vector $v$. Let $\chi\left(x_{\perp}\right)$ be the characteristic function of the window $W$ for the lattice points projected to $E_{\perp}$.

Prop 6: The Patterson function in $E_{\|}$at $x_{\|}=x-x_{\perp}$ is given by

$$
P^{t o t a l}\left(x_{\|}\right)=\sum_{v \in \Lambda} \delta\left(x_{\|}-v_{\|}\right) \int_{W} \chi\left(x_{\perp}\right) \chi\left(x_{\perp}-v_{\perp}\right) d x_{\perp}=\sum_{v \in \Lambda} \delta\left(x_{\|}-v_{\|}\right) P\left(v_{\perp}\right) .
$$

We shall put $P\left(v_{\|}\right)=P\left(v_{\perp}\right)$. A Patterson analysis within a plane perpendicular to the 5 fold axis 5 with a fixed shift vector $v_{\|}$parallel to this plane involves the following notions in $E_{\perp}$ : Consider the corresponding planar section of the triacontahedron with an intersection at $\eta \tau$ (5) along the 5 fold axis 5. The values of $\eta$ for the 11 selected planes are given in Table 1 both for the vertex points and for the centers of Bergman dodecahedra touching the vertex plane from below. The area of the planar section is proportional to the density $D(\eta)$ of vertex points. The terraces $2,5,10$ in this interpretation

are dense vertex planes. The terraces 2 and 7 have the highest, the terrace 6 has the lowest density of Bergman faces.

The Patterson function from Prop.4 is computed as follows: Shift the planar section by the vector $v_{\perp}$ parallel to itself. Compute the area of the intersection of the shifted and unshifted section. This area is proportional to the value of the Patterson function in $E_{\|}$at the point $v_{\|}$. The value of $P(0)$ is proportional to the density of points. We may also normalize by plotting $P\left(v_{\|}\right) / P(0)$.

In a circle approximation we proceed as follows:

We use the exact area $F(\eta)$ of a planar section of the triacontahedron as a function of $\eta$.

For any fixed value of $\eta$ we compute the radius $r(\eta)$ of a circle with the same area as the section, hence

$$
r(\eta):=\sqrt{\pi^{-1} F(\eta)} .
$$


This amounts to replacing the triacontahedron by a rotational surface whose circular areas (and hence density values) for any $\eta$ are equal to the ones of the triacontahedron. Then we approximate the Patterson function by using these circles instead of the polygonal sections as functions of $\eta$. In this approximation, the Patterson function only depends on $\left|v_{\perp}\right|$.

The Patterson function $P$ has a smooth behaviour in terms of the two variables $\eta$ and $v_{\perp}$. This expression is shown in Fig.11. For $v_{\perp}=0$ it reduces to the function $F(\eta)$ and for $v_{\perp}=2 r(0)$ it must go to zero.

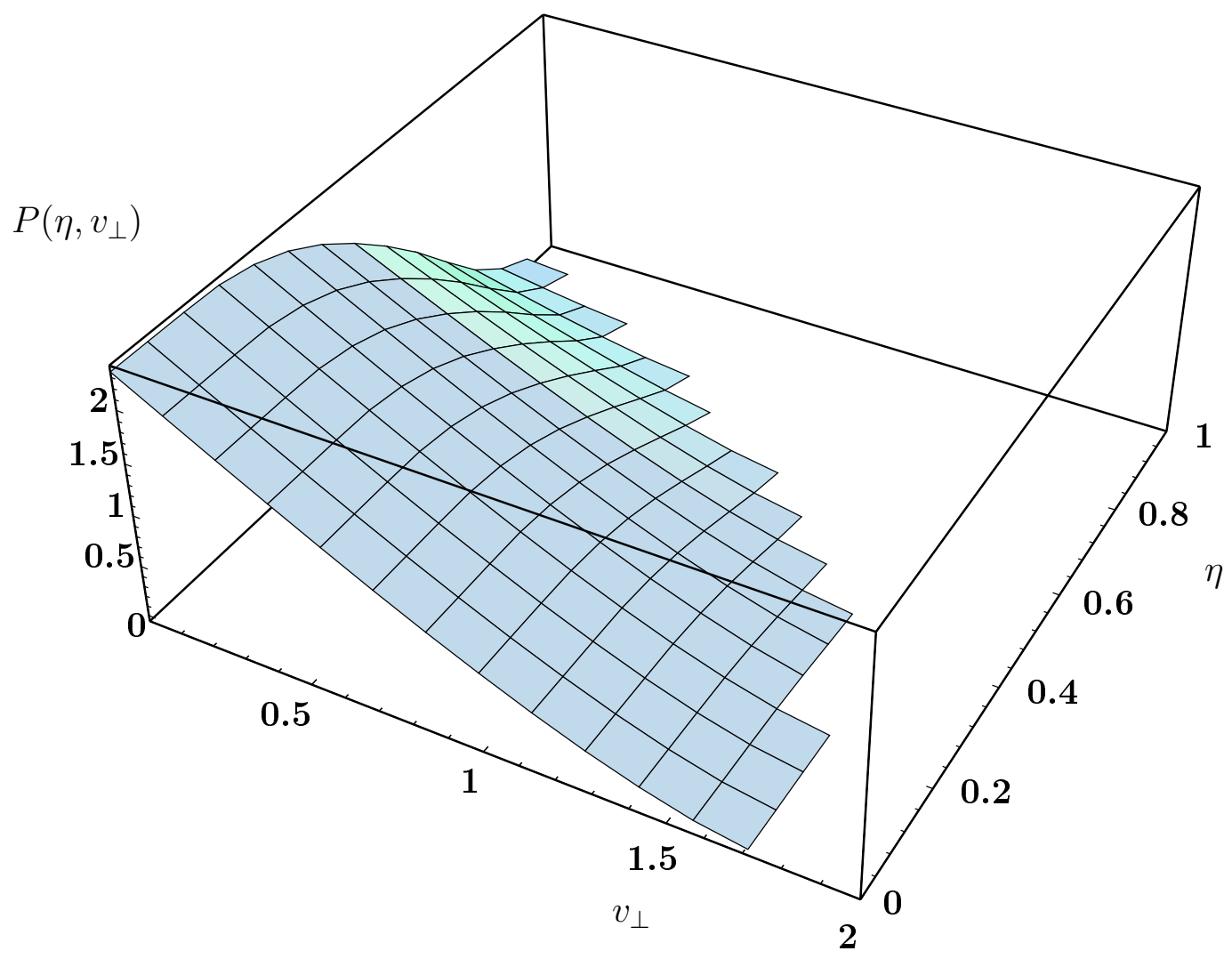

Fig.11 The Patterson function $P\left(\eta, v_{\perp}\right)$. For $v_{\perp}=0$ it reduces to $F(\eta)$.

For the plane number $16 \rightarrow 8$, we give in Figs. 12-14 the values of the Patterson function $P$ for $\eta_{1}, \eta_{2}, \eta_{3}$, represented by areas of circles, as a function of the 11 points $v_{\|}$selected in a plane as in $[15$. 

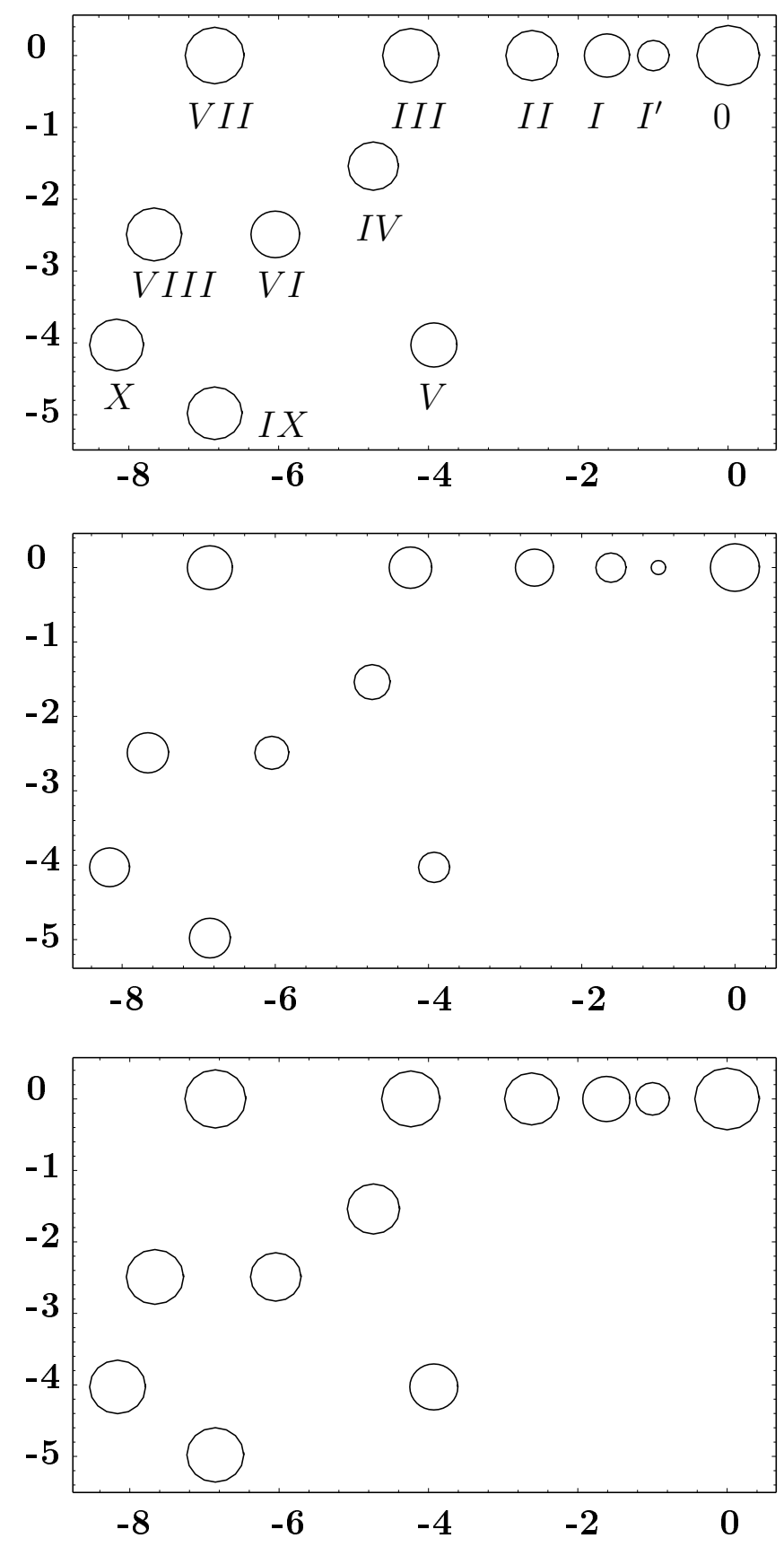

Figs.12-14. The Patterson function $P$ corresponding to the values $N=16 \rightarrow$ 8 for the values $\eta_{1}, \eta_{2}, \eta_{3}$ from Table 1 , represented by the area of circles in a plane. The upper right-hand circle stands for $0: v_{\|}=0$, the first point $I^{\prime}$ to its left is at a distance $\left|v_{\|}\right|=7.78 \AA$ the others are labelled by roman numbers $I \ldots X$ in correspondence to 15 . 
In Figs.15-17 we present the same values as a function of the 10 roman numbers which label peak positions $v_{\|}$. It can be seen that $P\left(\eta_{2}\right)$ yields the lowest density and the strongest relative variation, $P\left(\eta_{1}\right)$ and $P\left(\eta_{3}\right)$ are very similar, but the latter yields the highest density.
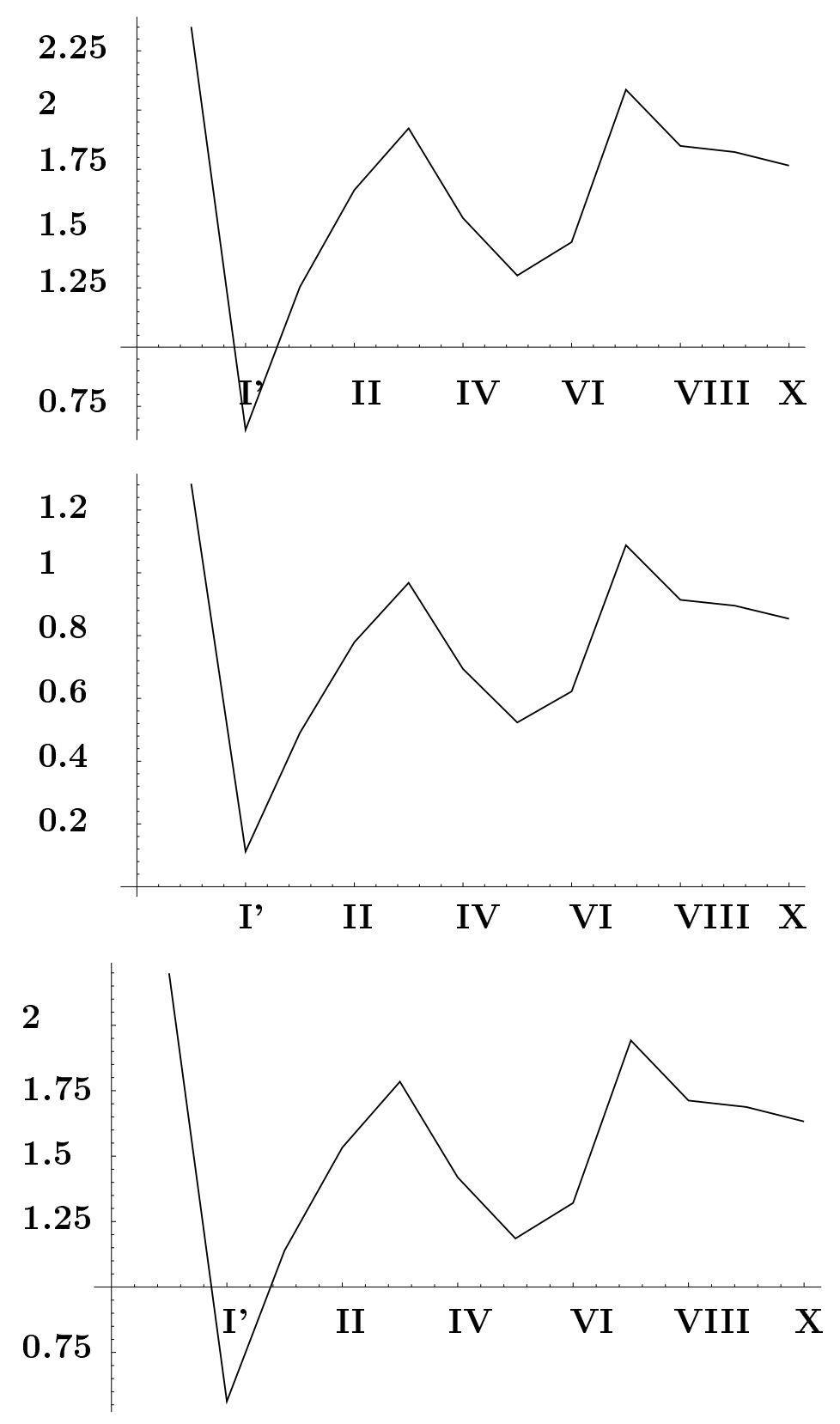

Figs.15-17. Patterson function $P$ as in the previous Figure as a function of the roman indices $I^{\prime}, I, \ldots X$ for the three values $\eta_{1}, \eta_{2}, \eta_{3}$ from top to bottom. 
We can now compare the three different models (i,ii,iii) of the terrace structure given in subsection 3.1 in terms of the Patterson data for terrace 8. Model (i) yields the lowest value of the Patterson data for the Bergman top faces, along with a larger value for the vertex points. Models (ii) and (iii) give the same larger values of the Patterson function for the small or the large top pentagons, but differ in the presence or absence of vertex points respectively. All three models are compatible with the qualitative set of experimental Patterson data 15.

\section{Conclusion.}

We analyze a tiling model for the surface structure of i- $A l_{68} P d_{23} M n_{9}$ quasicrystals perpendicular to a 5 fold axis. The surfaces are interpreted as terminations in atomic planes from the bulk tiling model decorated by Bergman clusters. The model is analyzed in a window approach. The quasiperiodic relations between planes occupied by atoms are rigorously and quantitatively derived from the bulk model. Sequences of planes are shown to be generated and connected by infinite Fibonacci lines running through the tiling. Both the density and the Patterson data for different types of atomic positions are computed in closed form and shown to vary strongly between the planes.

In the planes we consider vertex positions of the tiling and pentagonal positions arising from faces or top cuts through the Bergman clusters. Changes of their correlation between the planes are analyzed in three alternative models. The pentagonal holes observed in [15] admit an interpretation in terms of these pentagons. If they arise from the Bergman top pentagons, their observed larger size must reflect a local reconstruction of the surface. If they arise from the Bergman top cut pentagons, they agree with the observed size. The geometry, the spacing, the density and the Patterson functions are computed from the bulk model. The sequence and spacing of terraces and the available Patterson data from [15] are well reproduced. The observed Fibonacci string of planes yields information on the density of atomic positions. The observed size of the terraces shows some correlation with the model structure in the planes.

More detailed experimental studies of the terrace structure in i-quasicrystals are suggested. 


\section{Acknowledgement.}

The authors would like to thank Tobias Kramer for substantial help in the computation and presentation of data.

\section{References}

[1] Baake M, Kramer P, Schlottmann M and Zeidler D, Int. J. Mod. Phys. B4 (1990) 2217-67

[2] de Boissieu M, Stephens P, Boudard M, Janot C, Chapman D L and Audier M, J. Phys. Condens. Matter 6 (1994) 10725

[3] Ebert Ph, Yue F, and Urban K, Phys. Rev. B 57 (1998) 2821

[4] Elser V, Phil. Mag. B73 (1996) 641

[5] Gierer M, Van Hove M A, Goldman A I, Shen Z, Chang S-L, Jenks C-M, Zhang C-M, Thiel P A, Phys. Rev. Lett.78 (1997) 467

[6] Gierer M, Van Hove M A, Goldman A I, Shen Z, Chang S-L, Pinhero P J, Jenks C M, Anderegg J W, Zhang C-M, Thiel P A, Phys. Rev.B 57 (1998) 7628

[7] Katz A and Gratias D, in: Proc. 5th Int. Conf. on Quasicrystals, eds. C Janot and R Mosseri, World Scientific, Singapore 1995, pp. 164-167

[8] Kepler J (1619) Harmonice Mundi, Germ. Transl. Weltharmonik by M Caspar, p. 74, Oldenbourg, Munich 1973

[9] Kramer P and Neri R, Acta Cryst. A 40 (1984), 580-587

[10] Kramer P, Papadopolos Z, Zeidler D, Symmetries of Icosahedral Quasicrystals, in: Symmetry in Science V, eds B. Gruber, L. C. Biedenharn and H. D. Doebner, Plenum, New York (1991), 395-427

[11] Kramer P, Papadopolos Z and Liebermeister W, in: Proc. 6th Int. Conf. on Quasicrystals, eds. S. Takeuchi and T. Fujiwara, World Scientific, Singapore (1998), 71-76

Kramer P, Papadopolos Z and Liebermeister W, Atomic Positions for the Icosahedral F-Phase Tiling in: Proc. Aperiodic '97, eds.

[12] Kasner G, Papadopolos Z, Kramer P and Bürgler D E, submitted for publication 
[13] Papadopolos Z, Kramer P and Zeidler D, J. Non-Cryst. Solids 153,154 (1993), 215-220

[14] Moody R V, Meyer Sets and Their Duals, in: The Mathematics of LongRange Aperiodic Order, Ed. R. V. Moody, Kluwer, Dordrecht 1997, pp. 403-441

[15] Schaub T M, Bürgler D E, Güntherodt H-J, and Suck J B, Phys.Rev.Lett. 73 (1994) 1255-8, Schaub T M , Bürgler D E, Güntherodt H-J, Suck J B and Audier M Appl. Phys. A 61 (1995) 491-501 